\title{
Optical Identification of He White Dwarfs Orbiting Four Millisecond Pulsars in the Globular Cluster 47 Tucanae $^{1}$
}

\author{
M. Cadelano ${ }^{1,2}$, C. Pallanca ${ }^{1}$, F. R. Ferraro ${ }^{1}$, M. Salaris ${ }^{3}$, E. Dalessandro ${ }^{1}$, \\ B. Lanzoni ${ }^{1}$, P. C. C. Freire ${ }^{4}$ \\ 1 Dipartimento di Fisica e Astronomia, Università di Bologna, Viale Berti Pichat 6/2, \\ I-40127 Bologna, Italy \\ 2 INAF - Osservatorio Astronomico di Bologna, via Ranzani 1, I-40127 Bologna, Italy \\ 3 Astrophysics Research Institute, Liverpool John Moores University, IC2, Liverpool \\ Science Park, 146 Brownlow Hill, Liverpool L3 5RF, UK \\ 4 Max-Planck-Institute für Radioastronomie, D-53121 Bonn, Germany
}

\begin{abstract}
We used ultra-deep UV observations obtained with the Hubble Space Telescope to search for optical companions to binary millisecond pulsars (MSPs) in the globular cluster 47 Tucanae. We identified four new counterparts (to MSPs 47TucQ, 47TucS, 47TucT and 47TucY) and confirmed those already known (to MSPs 47TucU and 47TucW). In the color magnitude diagram, the detected companions are located in a region between the main sequence and the $\mathrm{CO}$ white dwarf cooling sequences, consistent with the cooling tracks of He white dwarfs of mass between $0.15 \mathrm{M}_{\odot}$ and $0.20 \mathrm{M}_{\odot}$. For each identified companion, mass, cooling age, temperature and pulsar mass (as a function of the inclination angle) have been derived and discussed. For 47TucU we also found that the past accretion history likely proceeded in a sub-Eddington rate. The companion to the redback $47 \mathrm{TucW}$ is confirmed to be a non degenerate star, with properties particularly similar to those observed for black widow systems. Two stars have been identified within the $2 \sigma$ astrometric uncertainty from the radio positions of $47 \mathrm{TucH}$ and 47TucI, but the available data prevent us from firmly assessing whether they are the true companions of these two MSPs.
\end{abstract}

Subject headings: Pulsars: Individual: J0024-7204H, J0024-7204I, J0024-7204Q, J0024-7204S, J0024-7204T, J0024-7203U, J0024-7204Y, Globular clusters: Individual: 47 Tucanae (NGC 104), Techniques: photometric

\footnotetext{
${ }^{1}$ Based on observations collected with the NASA/ESA HST (Prop. 12950), obtained at the Space Telescope Science Institute, which is operated by AURA, Inc., under NASA contract NAS5-26555.
} 


\section{INTRODUCTION}

Millisecond pulsars (MSPs) are rapidly spinning neutron stars (NSs) formed in a binary system where a slowly rotating NS is spun up through mass accretion from an evolving companion star. The recycling process is usually observed in the low mass X-ray binary systems, which are commonly considered as the MSP progenitors (Alpar et al. 1982; Bhattacharya \& van den Heuvel 1991; Papitto et al. 2013). Indeed, when the mass accretion rate decreases and the NS is sufficiently recycled, the rotation-powered emission is switched on and the system is observable as a MSP in the radio band. These processes usually lead to a deep transformation of the companion star which can transit through a highly perturbed evolutionary phase (possibly like MSP-A in NGC 6397; Ferraro et al. 2001a), before reaching the final stage of a (possibly He) white dwarf (WD; e.g. MSP-A in NGC 6752; Ferraro et al. 2003a). Each step of this evolution corresponds to objects characterized by different properties, that, in the optical bands, are imprinted in the observable features of the companion star.

According to the canonical scenario, the majority of binary MSPs have low-mass He WD companions (see, e.g., van Kerkwijk et al. 2005). However, recent pulsar (PSR) searches have considerably increased the number of non-canonical systems, especially the so-called "black widows" and "redbacks": ultra-compact binary systems (P presence of radio eclipses suggests the presence of ionized material ablated from a bloated companion star because of the energy injected by the PSR (Ruderman et al. 1989; Ray et al. 2012; Roberts 2013). Redback companion stars usually have masses of $0.1-0.5 \mathrm{M}_{\odot}$, while black widow companions are much less massive $\left(\mathrm{M}<0.1 \mathrm{M}_{\odot}\right)$. Such a small value could be due to vaporization from the strong MSP radiation and relativistic wind. The physical

mechanisms bringing to one or the other system are still debated. Simulations by Chen et al. (2013) show that redbacks and black widows are the outcome of different evolutionary paths, where the PSR irradiation efficiency is the discriminant factor. At odds with these results, the simulations by Benvenuto et al. (2014) show that the evolution of redbacks is bifurcated, with some of them evolving into black widows, and the others producing canonical He WD systems. Possibly, the progressive evaporation of the black widow companions could lead to the total disruption of the star and then to the formation of isolated MSPs. Interestingly, in recent years several connections between low mass X-ray binaries and redbacks have been found, especially with the discovery of systems transitioning from one state to the other (see Archibald et al. 2009; Papitto et al. 2013; Bassa et al. 2014).

Although the Galaxy is $\sim 100$ times more massive than the entire Galactic globular cluster (GC) system, about $40 \%$ of the known MSP population is found in GCs. Such an over-abundance is indicative of a strongly enhanced dynamical activity in these 
dense stellar systems, which promotes the formation of a conspicuous number of exotic objects, such as blue straggler stars, X-ray binaries, cataclysmic variables and MSPs (Bailyn 1992; Cool et al. 1995; Ferraro et al. 1995, 2001b, 2015a; Grindlay et al. 2002; Pooley et al. 2003; Ransom et al. 2005), which can be used to probe the complex interplay between dynamics and stellar evolution (e.g. Goodman \& Hut 1989; Hut et al. 1992; Phinnev 1992; Possenti et al. 2003; Ferraro et al. 2003b, 2009, 2012, 2015b; Verbunt \& Freire 2014). In this respect, the study of optical companions to binary MSPs in GCs is of outmost importance, since it opens the possibility to get insights on the impact of dynamical interactions (which are particularly frequent in dense environments) on MSP and stellar evolution, e.g., favoring binary formation (through tidal captures), binary shrinking (through fly-by) and consequent mass transfer activity, as well as exchange interactions able to substitute the original companion that recycled the pulsar, with a new, more or less perturbed, star (see e.g. Rasio et al. 2000; King et al. 2003; Ferraro et al. 2003c; Sabbi et al. 2003a.b; Mucciarelli et al. 2013; Benacquista \& Downing 2013). Moreover, in the case of WD companions, it is possible to estimate the masses and cooling ages of the systems by the direct comparison of their properties with stellar evolutionary models (see e.g. Ferraro et al. 2003a; Pallanca et al. 2013a), while accurate mass measurements require spectroscopical techniques (e.g. van Kerkwijk et al. 1996; Bassa et al. 2006; Antoniadis et al. 2012, 2013). In the case of MSPs in GCs, these constraints benefit from the known GC distance and optical extinction, thus reducing the uncertainties on the estimated quantities with respect to the case of MSPS in the Galactic field. The derived companion masses can be combined with radio timing parameters to estimate the PSR masses, thus allowing general relativity test (e.g. Freire et al. 2012) and fundamental physics studies, as the determination of the equation of state of ultra dense matter (Lattimer \& Prakash 2001). On the other hand, the derived cooling ages are a more appropriate measurement of the system age with respect to the characteristic PSR ages derived from radio timing (Tauris et al. 2012a; Tauris 2012b). The correct determination of MSP ages is an important tool to study the spin evolution and to constrain the physics of the recycling phases (see e.g. van Kerkwijk et al. 2005, and references therein).

Despite their importance, the identification of MSP optical companions is challenging in crowded stellar systems like GCs. Only ten companions have been discovered so far in GCs. Three companions are He WDs (see Edmonds et al. 2001; Ferraro et al. 2003a; Sigurdsson et al. 2003; Bassa et al. 2003, 2004), as expected from the canonical formation scenario, five are redbacks companions (see Ferraro et al. 2001a; Edmonds et al. 2002; Cocozza et al. 2008; Pallanca et al. 2010, 2013b) and two are black widow companions (Pallanca et al. 2014a; Cadelano et al. 2015).

The GC 47 Tucanae, located at a distance of about $4.5 \mathrm{kpc}$ from the Sun, hosts the largest population of MSPs after Terzan 5 (Freire et al. 2003; Ransom et al. 2005). Indeed, 
23 radio MSPs have been discovered so far, 14 of which are located in binary systems. Here we report the identification and the properties of four new MSP companions in 47 Tucanae and we present the follow-up study of two previously known companions. In Table 1 we report the main radio timing properties of the analyzed objects, which are useful in the following discussions. All the identified companions are likely canonical MSPs, except one which is a redback system.

In Section 2 we present the used photometric dataset and the identification of the MSP optical counterparts. In Section 3 we discuss in detail the properties of each companion. Finally, in Section 4, we summarize our results.

\section{OPTICAL PHOTOMETRY OF THE STAR CLUSTER}

\subsection{Observations and data analysis}

In the present study the identification of the MSP companions has been performed through an ultra-deep, high resolution, photometric dataset acquired under GO 12950 (P.I: Heinke) with the UVIS camera of the Wide Field Camera 3 (WFC3) mounted on the Hubble Space Telescope (HST). The dataset consists of 8 images in the F390W filter, with exposure times of $567-590 \mathrm{~s}$, and 24 images in the LP F300X filter, with exposure times of $604-609 \mathrm{~s}$.

The standard photometric analysis (see Dalessandro et al. 2008a, b) has been performed on the "flt" images, which are corrected for flat field, bias and dark counts. These images have been further corrected for "Pixel-Area-Map"3 with standard IRAF procedures. By using the DAOPHOT II packages (Stetson 1987), we performed an accurate photometric analysis of each image. First of all, we modeled a spatially varying Point Spread Function (PSF) by using a sample of $\sim 200$ bright but not saturated stars. The model has been chosen on the basis of a $\chi^{2}$ test and, in every image, the best fit is provided by a Moffat function (Moffat 1969). Then we performed a source detection analysis, setting a $3 \sigma$ detection limit, where $\sigma$ is the standard deviation of the measured background. Once a list of stars was obtained, we performed a PSF-fitting in each image using the ALLSTAR routine. In the resulting catalog we included only objects present at least in half the images for each filter. Then, this catalog has been further processed with the ALLFRAME routine. For each star, we homogenized the magnitudes estimated in different images, and their weighted mean and

\footnotetext{
${ }^{2}$ Please visit http://www.naic.edu/ pfreire/GCpsr.html, for a complete list of the main radio timing properties of MSPs in GCs.

${ }^{3}$ For more details see the WFC3 Data Handbook.
} 
standard deviation have been finally adopted as the star mean magnitude and its related photometric error (see Ferraro et al. 1991, 1992). However, in order to perform variability studies, for each source we also kept the homogenized magnitude measured in each frame in both filters. Then, instrumental magnitudes have been calibrated to the VEGAMAG system by using the zero points quoted in the WFC3 Data Handbook and by performing aperture corrections.

\subsection{Astrometry}

Since the WFC3 images suffer from geometric distortions, we corrected the instrumental positions (x,y) following Bellini et al. (2011). In order to transform the instrumental positions into the absolute astrometric system $(\alpha, \delta)$, we used, first of all, the wide field catalog presented in Ferraro et al. (2004). Its astrometric solution has been improved by crosscorrelation 4 with the UCAC4 astrometric standard catalog (Zacharias et al. 2013; $\sim 4600$ stars have been found in common between the two datasets). The latter is based on the International Celestial Reference System, thus allowing a more appropriate comparison with the MSP positions derived from timing using solar system ephemerides (which are referenced to the same system). The newly-astrometrized wide field catalog has then been used as a secondary reference frame to astrometrize the WFC3 data set, by means of $\sim 22000$ stars in common. The resulting $1 \sigma$ astrometric uncertainty is $0.10^{\prime \prime}$ and $0.11^{\prime \prime}$ in $\alpha$ and $\delta$, respectively. Thus the final total astrometric uncertainty is $\sim 0.15^{\prime \prime}$. Unfortunately, there are only few stars in common between the WFC3 and the UCAC4 catalogs, since the latter does not cover the cluster central regions. This prevented a direct cross-correlation between the two catalogs and thus we could not take into account the stellar proper motions between the two observation epochs, which would have reduced the astrometric uncertainty.

\subsection{Identification of the MSP companions}

First of all, in order to search for the companions to the MSPs in 47 Tucanae, we checked the precision of our astrometric solution re-identifying the two companion stars

already known in the cluster (see Edmonds et al. 2001, 2002). To this aim, we performed a

\footnotetext{
${ }^{4}$ We used CataXcorr, a code which is specifically developed to perform accurate astrometric solutions. It has been developed by P. Montegriffo at INAF- Osservatorio Astronomico di Bologna. This package is available at http://davide2.bo.astro.it/ paolo/Main/CataPack.html, and has been successfully used in a large number of papers by our group in the past 10 years.
} 
detailed analysis of all the detectable objects within a $5^{\prime \prime} \times 5^{\prime \prime}$ wide region centered on the nominal position of each MSP. The companion to 47TucU (COM-47TucU; hereafter all the companions will be named as COM-47Tuc followed by the letter of the respective MSP) and 47 TucW have been re-identified in stellar sources located at $0.06^{\prime \prime}$ from the MSP nominal positions. Both the identifications turn out to be largely within our astrometric uncertainty, thus confirming the accuracy of the adopted astrometric solution. The finding charts of these two reference objects are shown in Figure 1.

Following the same procedure, we searched for the companions to all the other MSPs with a known position (Freire et al. 2003, Freire et al. 2015, in preparation). Stars located within the $2 \sigma$ uncertainty from the pulsar position have been considered as possibile counterparts. Four companions (to 47TucQ, 47TucS, 47TucT and 47TucY) have been identified on the basis of their positional coincidence (all of them are located at a distance $\leq 0.06^{\prime \prime}$ from the nominal radio position) and of their position in the color magnitude diagram (CMD). Two faint stars have been detected also within the $2 \sigma$ uncertainty circle from 47TucI and $47 \mathrm{TucH}$. However, their distances $\left(0.15^{\prime \prime}\right.$ and $0.24^{\prime \prime}$ respectively) from the pulsar radio positions are significantly larger than in all the other cases, thus casting doubts about these objects being the true optical counterparts (see more discussion in Section 3.2). The finding charts of all these objects are shown in Figure 1 and their main photometric properties are reported in Table 2. Their location in the cluster CMD is shown in Figure 2, where only the stars with a sharpness parameter $|\mathrm{sh}| \leq 0.05$ are plotted. As can be seen, with the exception of the candidate companion to 47TucI, all the newly identified counterparts are located in the region where He WDs are expected, although the candidate companion to 47TucH could be compatible also with the CO WD cooling sequence (see Section 3.2). Since the radio timing properties suggest that these systems are the product of the canonical recycling scenario, their location along the He WD cooling sequences guarantees their connection with the MSPs. Note in fact that the probability of a chance coincidence with another He WD is extremely low $(\mathrm{P} \approx 0.1 \%) 6$, since these objects can only be the product of the late stage of the evolution of exotic objects like, for example, MSPs and cataclysmic variables. The candidate companion to $47 \mathrm{TucI}$ is instead a main sequence-like object, and its properties will be briefly discussed in Section 3.2. As concerns the previously known companions,

\footnotetext{
${ }^{5}$ The sharpness parameter is a DAOPHOT II output that quantifies the stellar-like structure of each object fitted with the PSF model. See the User Manual for more details.

${ }^{6}$ The chance coincidence probability has been evaluated predicting the number He WD expected within a radius equal to the $2 \sigma$ astrometric uncertainty. We derived the He WD density by direct counting of the all objects located among the cooling tracks (see Section 3.1 and Figure 3) and dividing this number by the size of the WFC3 field of view. Please note that even including all the stars of the catalog with sharpness $|\mathrm{sh}|>0.05$, the chance probability remains $\lesssim 0.5 \%$.
} 
COM-47TucU is also located along the He WD sequence, while the redback COM-47TucW is located in an anomalous region between the main sequence and the WD cooling sequence (see Section 3.4).

With the exception of the COM-47TucW, no significant variability related to the orbital period has been detected. For 47TucU, 47TucY and 47TucW (see Section 3.4) the observations sample a significant fraction of the orbital period. Instead, for the other systems (with orbital periods longer than 1 day) the coverage is too poor to allow any appropriate variability analysis. However, a strong magnitude modulation, as the one observed for non degenerate companions (see e.g. Stappers et al. 1999; Edmonds et al. 2002; Revnolds et al. 2007; Pallanca et al. 2010; Romani \& Shaw 2011; Pallanca et al. 2014a; Cadelano et al. 2015), is not expected and usually not observed for degenerate objects, since the flux enhancement due to re-heating of the companion star by the PSR emitted energy is negligible.

\section{DISCUSSION}

\subsection{The physical properties of the He WD companions}

In order to constrain the main properties of the He WD companions, we have compared the position of each candidate in the CMD with a set of He WD cooling tracks computed by Althaus et al. (2013). These models span a mass range from $0.15 \mathrm{M}_{\odot}$ to $0.43 \mathrm{M}_{\odot}$, spaced at about $0.005 M_{\odot}$ for masses between $0.15 \mathrm{M}_{\odot}$ to $0.19 \mathrm{M}_{\odot}$ and up to $0.07 \mathrm{M}_{\odot}$ for larger masses. We transformed the theoretical luminosities and temperatures into the absolute F300X and F390W magnitudes, by applying the bolometric corrections kindly provided by P. Bergeron (see Holberg \& Bergeron 2006; Bergeron et al. 2011). Then, the model absolute magnitudes have been transformed into the apparent ones by using the distance modulus $(m-M)_{0}=13.32 \pm 0.10$ (Ferraro et al. 1999)7. and the color excess $E(B-V)=0.04 \pm 0.02$ (Ferraro et al. 1999; Zoccali et al. 2001; Salaris et al. 2007) and extinction coefficients $A_{F 300 X} / A_{V}=1.77309, A_{F 390 W} / A_{V}=1.42879$ (Cardelli et al. 1989; O'Donnell 1994). Figure 3 shows the zoomed portion of the CMD in the WD region with a sample of cooling tracks for different masses overplotted. As can be seen, the range in mass of the models is large enough to properly sample the portion of the CMD where all the companions are located. Therefore we used this set of models to derive the combinations

7 Many literature works reported on different values of 47 Tucanae distance modulus (see, e.g. Woodley et al. 2012, and references therein). However, all these possibile values have only a minimal influence on our derived companion properties (e.g. the derived companion masses would vary of less than $\sim 7 \%$ for all the companion stars) 
of parameters (mass, cooling age and temperature) that simultaneously satisfy the observed photometric magnitudes in both the filters, also taking into account the uncertainties on the companion magnitudes, distance modulus and reddening. The best values have been evaluated with a simple $\chi^{2}$ statistic. In doing this, linear interpolations (for different masses but equal ages) among the tracks have been performed in order to have a tighter mass sampling. We assumed that each companion is located at the distance of 47 Tucanae 8 and it is affected by the same extinction 9 . Figure 4 shows, for each system, the combination of cooling age (left panel), temperature (central panel) and PSR mass (right panel) appropriate for the derived value of the companion mass. In particular, in each plot the right panel shows the results obtained for different values of the inclination angle and interesting constrains on each system can be drawn. For instance, by setting the inclination angle to $90^{\circ}$, the maximum PSR mass allowed from the inferred companion mass can be evaluated. Conversely, by assuming the minimum PSR mass equal to $1.17 \mathrm{M}_{\odot}$ (the lowest mass ever measured for a NS; Janssen et al. 2008), a conservative lower limit to the inclination angle can be derived. All these results are also summarized in Table 3 where the quoted uncertainties are the range of possibile values allowed by the comparison with the theoretical track 10 . Note that we are not analyzing here the cases of $47 \mathrm{TucH}$ and $47 \mathrm{TucI}$, which we will discuss in Section 3.2 .

As can be seen, all the companions have masses between $\sim 0.15 \mathrm{M}_{\odot}$ and $\sim 0.2 \mathrm{M}_{\odot}$. The derived ranges of ages are in agreement with the lower limits to the PSR characteristic ages reported in Table 1. The only exception is COM-47TucU, which is discussed below. In principle the mass of COM-47TucQ could be smaller than our best-fit value $\left(0.15 M_{\odot}\right)$, since not theoretical tracks for masses below this value are available. However, already a $0.15 M_{\odot}$ companion would imply an extremely low value of the PSR mass $\left(\lesssim 1 \mathrm{M}_{\odot}\right)$. This puzzling result could be partially explained with the difficulty of accurately determine the color of the optical counterpart, because of the presence of a very close bright object (see Figure 1).

Our results rule out a massive NS in the case of 47TucQ and 47TucT, while the possibility of $\mathrm{a} \sim 2 \mathrm{M}_{\odot}$ NS remains opened in the cases of 47TucS, 47TucU and 47TucY. However, Figure 4 shows that the PSR mass can be significantly reduced by assuming an intermediatelow inclination angle of the orbital plane. In any case these systems are worthy of future, especially spectroscopical, investigations.

\footnotetext{
${ }^{8}$ Even though Freire et al. (2001) measured distance offsets between the cluster MSPs, such differences are very small and can be neglected for our goals.

${ }^{9}$ The effects of differential reddening are negligible for our goals (see Milone et al. 2012a, b).

${ }^{10}$ The reader should be aware that the WD parameters should not be assumed at face value as perfectly correct but as estimations, since they are model dependent and could also suffer from some hardly quantifiable uncertainty linked to the bolometric corrections.
} 
We also compared our results with the theoretical predictions on the behavior of the orbital period as a function of the companion mass discussed in Tauris \& Savonije (1999). Such a model has been already empirically verified by Corongiu et al. (2012) and Bassa et al. (2006). As shown in Figure 6, where we also added the two He WD companions identified in NGC 6752 and M4 (see Ferraro et al. 2003a; Sigurdsson et al. 2003), our results are in reasonable agreement with the model. The analytical prediction seems to slightly overestimate the companion mass or to underestimate the system orbital period. However, this model is valid for binary systems with $0.18 \mathrm{M}_{\odot}<\mathrm{M}_{\mathrm{WD}}<0.45 \mathrm{M}_{\odot}$, thus only marginally representative of our sample, where most of the companions appear to be less massive than $0.18 \mathrm{M}_{\odot}$. More updated models (from Istrate et al. 2014; gray points in Figure 6) are in better agreement, although they are the results of simulations of donor stars with metallicity $\mathrm{Z}=0.02$, larger than that of 47 Tucanae (i.e. $\mathrm{Z}=0.008$, Lapenna et al. 2015).

The brightness of COM-47TucU allowed us to put tighter constraints to the system parameters with respect to the other objects. Both its mass and temperature are in excellent agreement with those reported in Edmonds et al. (2001), while our derived age $(\approx 0.9$ Gyr) turns out to be 0.3 Gyr larger than their estimate. Such a discrepancy could be due to the different theoretical models used. However, as already noticed by Edmonds et al. (2001), the cooling age is significantly lower than the characteristic age of 2.5 Gyr:11. This discrepancy should not alarm, since the PSR characteristic ages are based on many assumptions and large deviations from the companion cooling ages are commonly observed (see e.g. Lorimer \& Kramer 2012; Tauris et al. 2012a; Tauris 2012b). Using the WD age together with the intrinsic spin period derivative $\left(\dot{\mathrm{P}}=2.7 \pm 0.5 \times 10^{-20}\right.$; Freire et al., in preparation) and the actual spin period $(\mathrm{P} \approx 4.343 \mathrm{~ms})$, we evaluated a MSP birth spin period (the so-called equilibrium spin period) of $\mathrm{P}_{0} \approx 3.576 \mathrm{~ms}$. This value, combined with the surface magnetic field $\left(\mathrm{B} \approx 3.145 \times 10^{8} \mathrm{G}\right)$ and assuming a NS with a radius of $10 \mathrm{~km}$ and a canonical mass of $1.4 \mathrm{M}_{\odot}$, can be used to infer the typical accretion rate that reaccelerated the NS during the low mass X-ray binary phase. By using equation (8) of van den Heuvel (2009), we find that the system past accretion history likely proceeded at a sub-Eddington rate $\left(\dot{M} / \dot{M}_{E D D} \sim 0.02\right)$, as expected from the typical evolution of close binary systems with light donor stars (Tauris \& Savonije 1999; Istrate et al. 2014). Although the mass accretion rate strongly depends on the NS radius, the general result does not change assuming different radii or even different NS masses.

\footnotetext{
${ }^{11}$ This value is based on the estimate of the PSR spin-down rate from the orbital period derivative, which is precise enough for this system (Freire et al., in preparation).
} 


\subsection{Possible candidate companion stars}

As can be seen from Figures 2, 3] and 6, the possible companion to 47TucH appears to have properties quite different from those observed for the other companions, first of all its much larger distance from the MSP nominal position $\left(0.24^{\prime \prime}\right)$, which corresponds to almost twice our astrometric uncertainty. Moreover, following the procedure adopted in the previous section, we derived for this object a mass of $0.37 \pm 0.05 \mathrm{M}_{\odot}$. This value, combined with the binary system total mass of $1.61 \mathrm{M}_{\odot}$ (Freire et al. 2003), would imply a PSR mass of $\sim 1.25 \mathrm{M}_{\odot}$, a value slightly lower than expected for a recycled PSR, although still acceptable within the uncertainties. Its position in the CMD is compatible also with the CO WD cooling sequence, which would increase the probability of a chance coincidence to $\sim 2-3 \%$. Furthermore, at odds with the others objects, this candidate counterpart occupies an anomalous region in the orbital period companion mass plane shown in Figure 6. Although this anomaly could be real (since $47 \mathrm{TucH}$ has a large eccentricity, probably due to some kind of dynamical interaction), all these pieces of evidence suggest that the observed object is probably an isolated WD and the true companion star is still under the detection threshold (see Section 3.3).

A possible candidate companion to MSP 47TucI has been also detected (see Figure1 1 for the finding chart). This is a binary system with a short orbital period $(\sim 0.23$ days) and a very small eccentricity. From the PSR mass function, the companion is expected to be a very low mass star $\left(\mathrm{M}_{\mathrm{COM}} \sim 0.015 \mathrm{M}_{\odot}\right)$. The absence of radio eclipses, probably due to a low inclination angle, prevents its characterization as a black widow system. At $0.15^{\prime \prime}$ from the PSR position, we identified a star located at the faint-end of the cluster main sequence (see Figure 2 and Table 21). If we assume that the companion is a bloated star seen in a binary system with a low inclination angle, such a CMD position could be reasonable. However, the lack of any significant variability related to the orbital period prevents us from firmly associating this candidate to the MSP. In fact, the orbital period coverage of the F390W images is too poor, while the signal to noise ratio of the F300X data allows us to only infer that, in case of photometric variability, the maximum variation amplitude must be smaller than $\sim 0.8$ mag. We therefore conclude that it is more likely that the real companion star is still under the detection threshold. Indeed, the probability of a chance coincidence with a main sequence star is non negligible $(\sim 45-50 \%)$. We finally note that another object lies within the astrometric uncertainty circle, but its association with the MSP can be excluded, since it is a common CO WD, with properties incompatible with the MSP timing ephemeris. 


\subsection{Non detections}

No interesting counterparts have been identified for all the other known binary MSPs. These non-detections are likely due to companion stars still under the detection threshold (as in the case, e.g., of 47TucE and the black widow 47TucJ), or to the severe crowding conditions of the area surrounding the MSP positions (as in the case of the black widows $47 \mathrm{TucO}$ and $47 \mathrm{TucR}$ ). No search could be performed for 47TucX since its position is outside the field of view.

Considering that the companion to $47 \mathrm{TucJ}$ should be a non-degenerate object, its nondetection in UV passbands cannot be used to get useful information on its properties. Instead, the counterpart to 47TucE is expected to be He WD, which remains undetected down to our limiting magnitudes ( $\sim 25$ in the F300X filter and $\sim 25.5$ in the F390W filter). Hence, taking into account that the cooling age of $\mathrm{a} \sim 0.17 M_{\odot}$ WD at these detection thresholds is larger than the cluster age $(\sim 10-11$ Gyr; Gratton et al. 2003; Hansen et al. 2013), it is unlikely that this star has a mass similar to that estimated for the other companions. It is more probable that it is more massive than $0.2 M_{\odot}$ (corresponding to a faster cooling) and its cooling age is larger than 1 Gyr. The same should apply also to the case of $47 \mathrm{TucH}$ if its true companion is still under our detection limits (as suggested above). Interestingly, according to the theoretical relation of Tauris \& Savonije (1999) and the orbital periods of 47TucE and 47TucH ( $\sim 2.3$ and $\sim 2.4$ days, respectively), the companions to both these MPSs are indeed expected to have masses $\gtrsim 0.2 M_{\odot}$.

\subsection{The companion to the redback 47 TucW}

47 TucW is the only redback identified, so far, in 47 Tucanae. It is a binary MSP with a spin period of $2.35 \mathrm{~ms}$, an orbital period of $\sim 3.2 \mathrm{hr}$ (Camilo et al. 2000) and a companion mass of $\sim 0.15 \mathrm{M}_{\odot}$. The first optical identification of this system was presented in Edmonds et al. (2002), who suggested that the companion is a perturbed and non degenerate star with a light curve structure indicating a strong heating by the PSR flux. In Figure 7 we show, for both the filters, the light curves we obtained by folding our photometric measurements with the most updated radio timing ephemeris (Freire et al., in preparation). The zero orbital phase has been set at the PSR ascending node time12. As can be seen, in agreement with previous works (Edmonds et al. 2002; Bogdanov et al. 2005), the light curves present a single maximum-minimum structure, likely due to the heating by the PSR

\footnotetext{
${ }^{12}$ Please note that we are using a different formalism with respect to Edmonds et al. (2002).
} 
flux. Unfortunately, the star has been measured above the detection threshold only near its maximum luminosity. Nonetheless, modeling 13 the sinusoidal light curve, we found that, in both the filters, the companion spans $\sim 3.5$ magnitudes between the maximum and the derived minimum, in agreement with previous observations. The best fit-model is shown as a solid curve in Figure 7. Interestingly, the light curve structure is more similar to the ones observed for black widow than for redback companions, which usually, but not always, show a double minimum-maximum structure due to tidal deformation (see e.g. Ferraro et al. 2003a; Cocozza et al. 2008; Pallanca et al. 2010; Li et al. 2014). The CMD position of the companion during the maximum and at a mean phase (as derived by the adopted model) is shown in Figure 2, The system is located between the main sequence and the WD cooling sequence, where no normal stars are expected and thus suggesting a perturbed and strongly heated companion star. Again, at odds with other redback systems, this lies in a region more similar to that occupied by the two black widow companions identified so far in GCs (Pallanca 2014b, Cadelano et al., 2015). The X-ray counterpart to 47TucW shows a variability which is likely due to an intra-binary shock between the PSR wind and the matter lost by the companion (Bogdanov et al. 2005). Interestingly, as discussed by Bogdanov et al. (2006), the minimum of the X-ray light curve is displaced with respect to the optical one. Such a behavior has been also noticed for the black widow M71A (Cadelano et al. 2015), thus further strengthening the connection of this MSP with black widow systems. All this allow us to speculate that a scenario where $47 \mathrm{TucW}$ will evolve into a canonical MSP with a He WD companion (as in the case of MSP-A in NGC 6397; see Burderi et al. 2002) is somewhat unlikely, opening the possibility to an evolution toward the black widow stages. Indeed such an evolutionary path has been already suggested by the simulations of Benvenuto et al. (2014). The identification of new redback companions will shed light on this possibility.

\section{SUMMARY}

By using ultra-deep, high resolution UV WFC3/HST observations of 47 Tucanae, we identified the companions to four binary MSPs (47TucQ, 47TucS, 47TucT and 47TucY) and confirmed the two already known objects (COM-47TucU and COM-47TucW). The optical counterparts have coordinates compatible, within the errors, with the PSR nominal positions. In the CMD, all the objects are located in the He WD cooling sequence, as expected from the MSP canonical evolutionary scenario. The only exception is the companion to the redback system $47 \mathrm{TucW}$, which is located in an anomalous region between the main sequence and

\footnotetext{
${ }^{13}$ We used the "Graphical Analyzer for TIme Series", a software aimed at studying stellar variability phenomena, developed by Paolo Montegriffo at INAF-Osservatorio Astronomico di Bologna.
} 
the WD cooling sequence, suggesting that it is a low-mass MS star highly perturbed and heated by the PSR flux. We compared the observed CMD positions of the detected He WD companions with a set of cooling tracks and derived the companion main properties (as masses, cooling ages, temperatures) and also some constraints on the PSR masses. All the companion stars have masses between $\sim 0.15 \mathrm{M}_{\odot}$ and $\sim 0.20 \mathrm{M}_{\odot}$, and all the derived cooling ages are smaller than the cluster stellar population age. The orbital periods vs companion masses are in fair agreement with the evolutionary models of Tauris \& Savonije (1999) and Istrate et al. (2014). By combining the cooling age with the PSR spin down rate we found that the accretion history of 47 TucU likely proceeded at a sub-Eddington rate.

By taking into account our astrometric uncertainty $\left(0.15^{\prime \prime}\right)$, we also detected a star having a position marginally compatible with that of 47TucH. However, its photometric properties would imply a PSR mass lighter than expected for a recycled NS. Moreover, its position in the plane of orbital period vs. companion mass is in clear disagreement with the theoretical predictions. While this could be due to its high eccentricity, the object could be just a chance coincidence and further investigations are needed before confirming its association to 47TucH. A possibile counterpart to 47TucI has been also identified in a star located in a low luminosity region of the cluster main sequence. However its distance from the MSP position $\left(0.15^{\prime \prime}\right)$ and the absence of any optical variability related to the orbital period do not allow us to asses a clear connection with the binary system.

Finally we discussed how the properties of COM-47TucW are more similar to those usually observed for black widows than for redbacks, thus opening the possibility that this MSP could be the prototype of a redback evolving into a black widow system.

\section{Acknowledgements}

This research is part of the project Cosmic-Lab (http://www.cosmic-lab.eu) funded

by the European Research Council under contract ERC-2010-AdG-267675. The authors kindly thank P. Bergeron and S. Cassisi for the help with the cooling tracks and isochrones, and T. Tauris and A. Istrate for providing us with their simulation data. M.C. thanks A. Istrate for the useful discussion.

\section{REFERENCES}

Alpar, M. A., Cheng, A. F., Ruderman, M. A., \& Shaham, J. 1982, Nature, 300, 728

Althaus, L. G., Panei, J. A., Romero, A. D., et al. 2009, A\&A, 502, 207 
Althaus, L. G., Miller Bertolami, M. M., \& Córsico, A. H. 2013, A\&A, 557, A19

Antoniadis, J., van Kerkwijk, M. H., Koester, D., et al. 2012, MNRAS, 423, 3316

Antoniadis, J., Freire, P. C. C., Wex, N., et al. 2013, Science, 340, 448

Archibald, A. M., Stairs, I. H., Ransom, S. M., et al. 2009, Science, 324, 1411

Bailyn, C. D. 1992, ApJ, 392, 519

Bassa, C. G., Verbunt, F., van Kerkwijk, M. H., \& Homer, L. 2003, A\&A, 409, L31

Bassa, C., Pooley, D., Homer, L., et al. 2004, ApJ, 609, 755

Bassa, C. G., van Kerkwijk, M. H., Koester, D., \& Verbunt, F. 2006, A\&A, 456, 295

Bassa, C. G., Patruno, A., Hessels, J. W. T., et al. 2014, MNRAS, 441, 1825

Bellini, A., Anderson, J., \& Bedin, L. R. 2011, PASP, 123, 622

Benacquista, M. J., \& Downing, J. M. B. 2013, Living Reviews in Relativity, 16, 4

Benvenuto, O. G., De Vito, M. A., \& Horvath, J. E. 2014, ApJ, 786, LL7

Bergeron, P., Wesemael, F., Dufour, P., et al. 2011, ApJ, 737, 28

Bhattacharya, D., \& van den Heuvel, E. P. J. 1991, Phys. Rep., 203, 1

Bogdanov, S., Grindlay, J. E., \& van den Berg, M. 2005, ApJ, 630, 1029

Bogdanov, S., Grindlay, J. E., Heinke, C. O., et al. 2006, ApJ, 646, 1104

Burderi, L., D’Antona, F., \& Burgay, M. 2002, ApJ, 574, 325

Cadelano, M., Pallanca, C., Ferraro, F. R., et al. 2015, ApJ, 807, 91

Camilo, F., Lorimer, D. R., Freire, P., Lyne, A. G., \& Manchester, R. N. 2000, ApJ, 535, 975

Cardelli, J. A., Clayton, G. C., \& Mathis, J. S. 1989, ApJ, 345, 245

Chen, H.-L., Chen, X., Tauris, T. M., \& Han, Z. 2013, ApJ, 775, 27

Cocozza, G., Ferraro, F. R., Possenti, A., \& D’Amico, N. 2006, ApJ, 641, L129

Cocozza, G., Ferraro, F. R., Possenti, A., et al. 2008, ApJ, 679, L105 
Cool, A. M., Grindlay, J. E., Cohn, H. N., Lugger, P. M., \& Slavin, S. D. 1995, ApJ, 439, 695

Corongiu, A., Burgay, M., Possenti, A., et al. 2012, ApJ, 760, 100

Cutri, R. M., Skrutskie, M. F., van Dyk, S., et al. 2003, VizieR Online Data Catalog, 2246, 0

Edmonds, P. D., Gilliland, R. L., Camilo, F., Heinke, C. O., \& Grindlay, J. E. 2002, ApJ, 579,741

Ferraro, F. R., Clementini, G., Fusi Pecci, F., \& Buonanno, R. 1991, MNRAS, 252, 357

Ferraro, F. R., Clementini, G., Fusi Pecci, F., Sortino, R., \& Buonanno, R. 1992, MNRAS, 256,391

Ferraro, F. R., Fusi Pecci, F., \& Bellazzini, M. 1995, A\&A, 294, 80

Ferraro, F. R., Messineo, M., Fusi Pecci, F., et al. 1999, AJ, 118, 1738

Ferraro, F. R., Possenti, A., D’Amico, N., \& Sabbi, E. 2001a, ApJ, 561, L93

Ferraro, F. R., D’Amico, N., Possenti, A., Mignani, R. P., \& Paltrinieri, B. 2001b, ApJ, 561, 337

Ferraro, F. R., Possenti, A., Sabbi, E., \& D’Amico, N. 2003a, ApJ, 596, L211

Ferraro, F. R., Possenti, A., Sabbi, E., et al. 2003b, ApJ, 595, 179

Ferraro, F. R., Sabbi, E., Gratton, R., et al. 2003c, ApJ, 584, L13

Ferraro, F. R., Beccari, G., Rood, R. T., et al. 2004, ApJ, 603, 127

Ferraro, F. R., Dalessandro, E., Mucciarelli, A., et al. 2009, Nature, 462, 483

Ferraro, F. R., Lanzoni, B., Dalessandro, E., et al. 2012, Nature, 492, 393

Ferraro, F. R., Pallanca, C., Lanzoni, B., et al. 2015a, ApJ, 807, L1

Ferraro, F. R., Lanzoni, B., Dalessandro, E., Mucciarelli, A., \& Lovisi, L. 2015, Ecology of Blue Straggler StarsAstrophysics and Space Science Library, Volume 413. ISBN 978-3-662-44433-7. Springer-Verlag Berlin Heidelberg, 2015, p. 99, 99

Freire, P. C., Kramer, M., Lyne, A. G., et al. 2001, ApJ, 557, L105 
Freire, P. C., Camilo, F., Kramer, M., et al. 2003, MNRAS, 340, 1359

Freire, P. C. C., Wex, N., Esposito-Farèse, G., et al. 2012, MNRAS, 423, 3328

Goodman, J., \& Hut, P. 1989, Nature, 339, 40

Gratton, R. G., Bragaglia, A., Carretta, E., et al. 2003, A\&A, 408, 529

Dalessandro, E., Lanzoni, B., Ferraro, F. R., et al. 2008a, ApJ, 677, 1069

Dalessandro, E., Lanzoni, B., Ferraro, F. R., et al. 2008b, ApJ, 681, 311

Edmonds, P. D., Gilliland, R. L., Heinke, C. O., Grindlay, J. E., \& Camilo, F. 2001, ApJ, $557, \mathrm{~L} 57$

Edmonds, P. D., Gilliland, R. L., Camilo, F., Heinke, C. O., \& Grindlay, J. E. 2002, ApJ, 579, 741

Grindlay, J. E., Camilo, F., Heinke, C. O., et al. 2002, ApJ, 581, 470

Hansen, B. M. S., Kalirai, J. S., Anderson, J., et al. 2013, Nature, 500, 51

Harris, W. E. 2010, arXiv:1012.3224

Holberg, J. B., \& Bergeron, P. 2006, AJ, 132, 1221

Hut, P., McMillan, S., Goodman, J., et al. 1992, PASP, 104, 981

Istrate, A. G., Tauris, T. M., \& Langer, N. 2014, A\&A, 571, A45

Janssen, G. H., Stappers, B. W., Kramer, M., et al. 2008, A\&A, 490, 753

King, A. R., Davies, M. B., \& Beer, M. E. 2003, MNRAS, 345, 678

Lapenna, E., Origlia, L., Mucciarelli, A., et al. 2015, ApJ, 798, 23

Lattimer, J. M., \& Prakash, M. 2001, ApJ, 550, 426

Li, M., Halpern, J. P., \& Thorstensen, J. R. 2014, ApJ, 795, 115

Lorimer, D. R., \& Kramer, M. 2012, Handbook of Pulsar Astronomy, by D. R. Lorimer , M. Kramer, Cambridge, UK: Cambridge University Press, 2012,

Milone, A. P., Piotto, G., Bedin, L. R., et al. 2012, ApJ, 744, 58

Milone, A. P., Piotto, G., Bedin, L. R., et al. 2012, A\&A, 540, A16 
Moffat, A. F. J. 1969, A\&A, 3, 455

Mucciarelli, A., Salaris, M., Lanzoni, B., et al. 2013, ApJ, 772, L27

O’Donnell, J. E. 1994, ApJ, 422, 158

Papitto, A., Ferrigno, C., Bozzo, E., et al. 2013, Nature, 501, 517

Pallanca, C., Dalessandro, E., Ferraro, F. R., et al. 2010, ApJ, 725, 1165

Pallanca, C., Dalessandro, E., Ferraro, F. R., Lanzoni, B., \& Beccari, G. 2013, ApJ, 773, 122

Pallanca, C., Lanzoni, B., Dalessandro, E., et al. 2013, ApJ, 773, 127

Pallanca, C., Ransom, S. M., Ferraro, F. R., et al. 2014, arXiv:1409.1424

Pallanca, C. 2014, arXiv:1405.2898

Phinney, E. S. 1992, Royal Society of London Philosophical Transactions Series A, 341, 39

Pooley, D., Lewin, W. H. G., Anderson, S. F., et al. 2003, ApJ, 591, L131

Possenti, A., D’Amico, N., Manchester, R. N., et al. 2003, ApJ, 599, 475

Ransom, S. M., Hessels, J. W. T., Stairs, I. H., et al. 2005a, Science, 307, 892

Rasio, F. A., Pfahl, E. D., \& Rappaport, S. 2000, ApJ, 532, L47

Ray, P. S., Abdo, A. A., Parent, D., et al. 2012, arXiv:1205.3089

Reynolds, M. T., Callanan, P. J., Fruchter, A. S., et al. 2007, MNRAS, 379, 1117

Roberts, M. S. E. 2013, IAU Symposium, 291, 127

Romani, R. W., \& Shaw, M. S. 2011, ApJ, 743, L26

Ruderman, M., Shaham, J., Tavani, M., \& Eichler, D. 1989, ApJ, 343, 292

Sabbi, E., Gratton, R. G., Bragaglia, A., et al. 2003a, A\&A, 412, 829

Sabbi, E., Gratton, R., Ferraro, F. R., et al. 2003b, ApJ, 589, L41

Salaris, M., Held, E. V., Ortolani, S., Gullieuszik, M., \& Momany, Y. 2007, A\&A, 476, 243

Sigurdsson, S., Richer, H. B., Hansen, B. M., Stairs, I. H., \& Thorsett, S. E. 2003, Science, 301,193 
Stetson, P. B. 1987, PASP, 99, 191

Stappers, B. W., van Kerkwijk, M. H., Lane, B., \& Kulkarni, S. R. 1999, ApJ, 510, L45

Tauris, T. M., \& Savonije, G. J. 1999, A\&A, 350, 928

Tauris, T. M., Langer, N., \& Kramer, M. 2012, MNRAS, 425, 1601

Tauris, T. M. 2012, Science, 335, 561

van den Heuvel, E. P. J. 2009, Astrophysics and Space Science Library, 359, 125

van Kerkwijk, M. H., Bergeron, P., \& Kulkarni, S. R. 1996, ApJ, 467, L89

van Kerkwijk, M. H., Bassa, C. G., Jacoby, B. A., \& Jonker, P. G. 2005, Binary Radio Pulsars, 328, 357

Verbunt, F., \& Freire, P. C. C. 2014, A\&A, 561, A11

Woodley, K. A., Goldsbury, R., Kalirai, J. S., et al. 2012, AJ, 143, 50

Zacharias, N., Finch, C. T., Girard, T. M., et al. 2013, AJ, 145, 44

Zoccali, M., Renzini, A., Ortolani, S., et al. 2001, ApJ, 553, 733 
Table 1. Radio timing ephemeris of the analyzed MSPs

\begin{tabular}{ccccccc}
\hline \hline MSP & $\alpha(\mathrm{h} \mathrm{m} \mathrm{s})$ & $\delta\left({ }^{\circ}{ }^{\prime \prime}\right)$ & Offset $\left({ }^{\prime}\right)$ & $\mathrm{P}_{\mathrm{ORB}}(\mathrm{d})$ & $f\left(M_{\odot}\right)$ & $\tau_{\text {age }}(\mathrm{Gyrs})^{b}$ \\
\hline 47TucH & $00246.7014(3)$ & $-72046.795(1)$ & 0.77 & 2.36 & $1.927 \times 10^{-3}$ & $>0.93$ \\
\hline $47 \mathrm{TucI}$ & $00247.9330(3)$ & $-720439.669(1)$ & 0.29 & 0.23 & $1.156 \times 10^{-6}$ & $>0.23$ \\
\hline $47 \mathrm{TucQ}$ & $002416.4891(4)$ & $-720425.153(2)$ & 0.98 & 1.19 & $2.374 \times 10^{-3}$ & $>1.43$ \\
\hline $47 \mathrm{TucS}$ & $00243.9779(4)$ & $-720442.342(1)$ & 0.19 & 1.20 & $3.345 \times 10^{-4}$ & $>0.91$ \\
\hline $47 \mathrm{TucT}$ & $00248.548(2)$ & $-720438.926(7)$ & 0.34 & 1.13 & $2.030 \times 10^{-3}$ & $>0.32$ \\
\hline $47 \mathrm{TucU}$ & $00249.8351(2)$ & $-720359.6760(9)$ & 0.94 & 0.43 & $8.532 \times 10^{-4}$ & 2.5 \\
\hline $47 \mathrm{TucW}^{b}$ & $00246.059(1)$ & $-720449.084(2)$ & $0.08^{a}$ & $0.13^{a}$ & $8.77 \times 10^{-4}$ & $>1.15$ \\
\hline $47 \mathrm{TucY}^{b}$ & $00241.4023(3)$ & $-720441.837(1)$ & 0.37 & $0.52^{a}$ & $1.195 \times 10^{-3}$ & $>2.2$ \\
\hline \hline
\end{tabular}

Note. - From left to right: MSP name, position, offset from the GC center, orbital period, mass function and characteristic age. Numbers in parentheses are uncertainties in the last digits quoted. Reference: Freire et al. (2003).

${ }^{a}$ http://www.naic.edu/ pfreire/GCpsr.html.

${ }^{b} \mathrm{P}$. Freire et al. 2015, in preparation. 
Table 2. Optical properties of the companion stars

\begin{tabular}{lccccc}
\hline \hline \multicolumn{1}{c}{ Name } & $\alpha(\mathrm{h} \mathrm{m} \mathrm{s})$ & $\delta\left({ }^{\circ}{ }^{\prime \prime \prime}\right)$ & $\operatorname{dist}\left({ }^{\prime \prime}\right)$ & $\mathrm{m}_{\text {F300x }}$ & $\mathrm{m}_{\text {F390W }}$ \\
\hline COM-47TucQ & 002416.489 & -720425.209 & 0.04 & $23.19 \pm 0.02$ & $23.63 \pm 0.05$ \\
\hline COM-47TucS & 00243.977 & -720442.385 & 0.03 & $23.29 \pm 0.02$ & $23.80 \pm 0.05$ \\
\hline COM-47TucT & 00248.549 & -720438.965 & 0.04 & $23.07 \pm 0.02$ & $23.56 \pm 0.03$ \\
\hline COM-47TucU & 00249.835 & -720359.746 & 0.06 & $20.40 \pm 0.01$ & $20.85 \pm 0.03$ \\
\hline COM-47TucW & 00246.063 & -720449.133 & 0.06 & $24.28^{a}$ & $23.62^{a}$ \\
\hline COM-47TucY & 00241.401 & -720441.875 & 0.04 & $22.16 \pm 0.02$ & $22.69 \pm 0.04$ \\
\hline COM-47TucH? & 00246.755 & -72046.781 & 0.24 & $23.39 \pm 0.02$ & $24.25 \pm 0.05$ \\
\hline COM-47TucI? & 00247.953 & -720439.559 & 0.15 & $24.14 \pm 0.04$ & $22.43 \pm 0.03$ \\
\hline \hline
\end{tabular}

Note. - From left to right: MSP name, position, distance from the radio MSP nominal position, F300X and F390W magnitudes and the relatives uncertainties.

aThe values for COM-47TucW correspond to the mean magnitudes of the best-fit models (see Figure 7).

Table 3. Derived properties of the five MSPs with He WD companions

\begin{tabular}{lccccc}
\hline \hline Parameter & 47TucQ & 47TucS & 47TucT & 47TucU & 47TucY \\
\hline $\mathrm{M}_{\mathrm{COM}}\left(\mathrm{M}_{\odot}\right)$ & $\sim 0.15$ & $0.17_{-0.02}^{+0.03}$ & $0.16_{-0.01}^{+0.025}$ & $0.171_{-0.003}^{+0.002}$ & $0.17 \pm 0.02$ \\
\hline Age $(\mathrm{Gyrs})$ & $\sim 5.5$ & $6.4_{-6.0}^{+1.7}$ & $5.1_{-3.5}^{+0.9}$ & $0.88_{-0.06}^{+0.05}$ & $2.2_{-1.6}^{+1.0}$ \\
\hline $\mathrm{T}\left(10^{3} \mathrm{~K}\right)$ & $\sim 7.6$ & $8.1_{-0.7}^{+1.0}$ & $8.0_{-0.5}^{+0.6}$ & $11.9_{-0.5}^{+0.2}$ & $9.6_{-1.2}^{+0.5}$ \\
\hline $\mathrm{L}\left(10^{-3} \mathrm{~L}_{\odot}\right)$ & $\sim 9.5$ & $8.1_{-0.3}^{+1.0}$ & $10.0_{-0.6}^{+0.5}$ & $158_{-17}^{+7}$ & $23.0 \pm 5$ \\
\hline $\mathrm{M}_{\mathrm{PSR}}\left(\mathrm{M}_{\odot}\right)$ & $<1.57$ & $<4.69$ & $<1.58$ & $<2.30$ & $<2.22$ \\
\hline$i\left(^{\circ}\right)$ & $>58$ & $>26$ & $>57$ & $>42$ & $>45$ \\
\hline \hline
\end{tabular}

Note. - From top to bottom: companion mass, age, temperature, luminosity, PSR mass and inclination angle. 


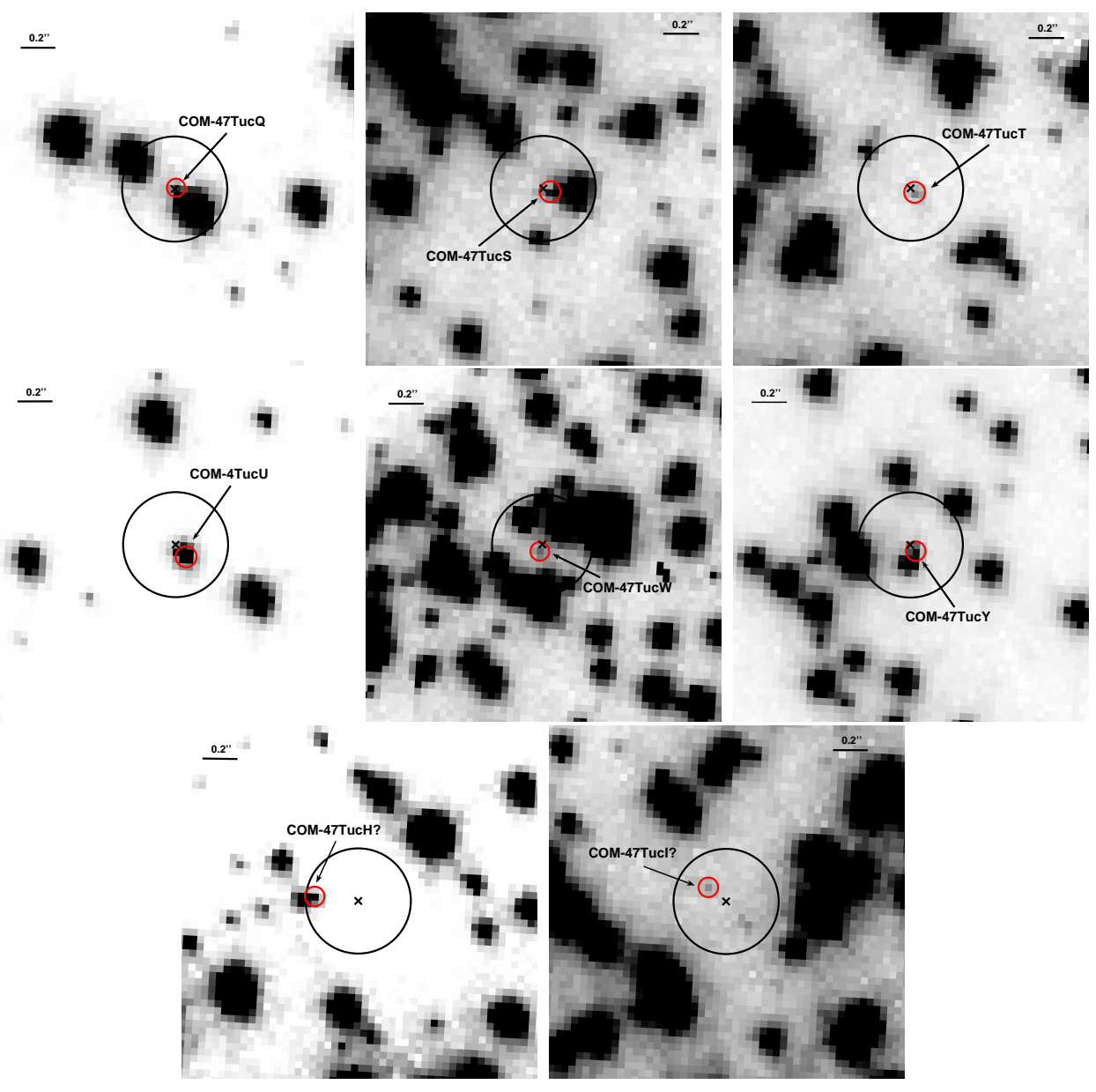

Fig. 1.- HST images of the $2^{\prime \prime} \times 2^{\prime \prime}$ region around the nominal position of the seven MSPs analyzed in this work. North is up and east is left. All the charts are obtained from a combinations of the available F300X images, with the exception of that of $47 \mathrm{TucW}$ that is from an image where the companion star is at its maximum luminosity. The black circles are centered on the radio PSR nominal position in the optical astrometric system and their radii are equal to our $2 \sigma$ astrometric uncertainty $\left(0.30^{\prime \prime}\right)$. The red circles mark the identified MSP companions. 


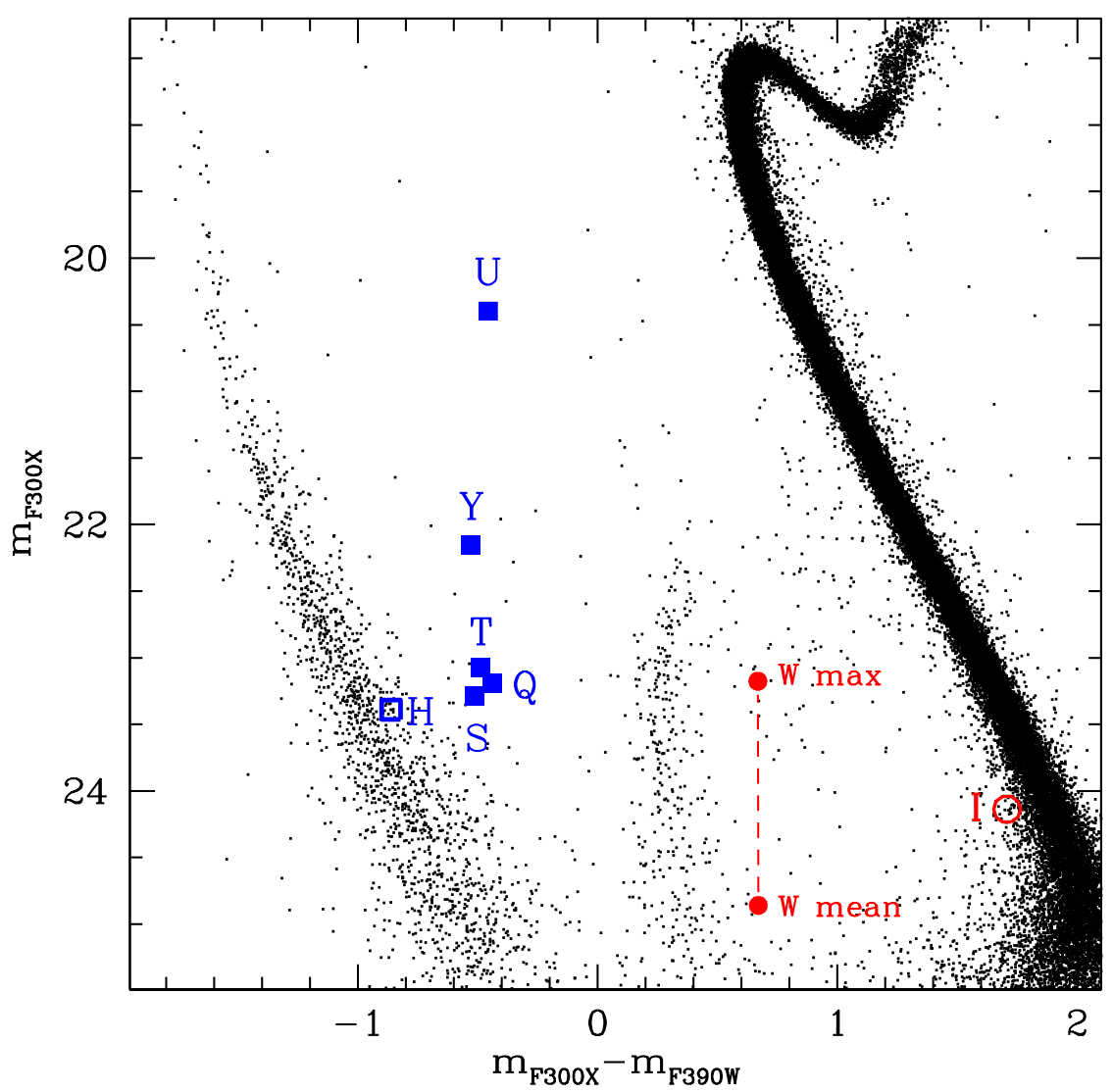

Fig. 2.- UV CMD of the GC 47 Tucanae. Only stars with sharpness parameter $|\operatorname{sh}| \leq$ 0.05 are plotted. The blue solid squares mark the companions to the canonical MSPs. The possible counterparts to $47 \mathrm{TucH}$ and $47 \mathrm{TucI}$ are plotted as an open square and circle respectively. Since COM-47TucW is a strongly variable object, we report its position at the maximum and mean luminosities, as derived by the best-fit models (see Section 3.4 and Figure 7). 


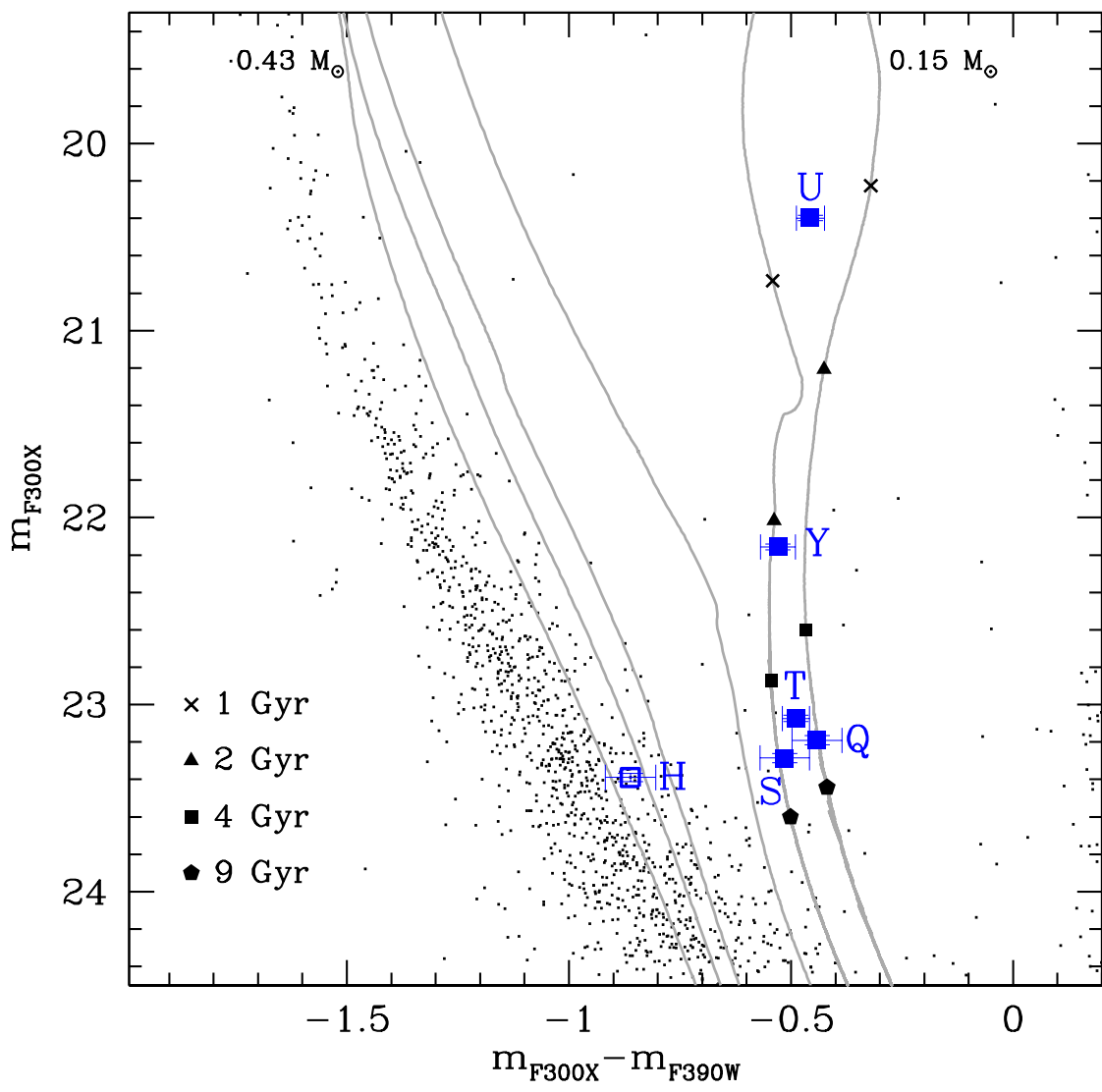

Fig. 3.- Same as in Figure 2, but zoomed into the WD region. The continuous curves are reference He WD cooling tracks for stars of $0.15 \mathrm{M}_{\odot}, 0.17 \mathrm{M}_{\odot}, 0.20 \mathrm{M}_{\odot}, 0.32 \mathrm{M}_{\odot}, 0.36 \mathrm{M}_{\odot}$ and $0.43 \mathrm{M}_{\odot}$ (from right lo left). For the two rightmost tracks, points at 1,2,4 and 9 Gyrs have been marked with different symbols. The photometric errors of the companion stars are also drawn. 

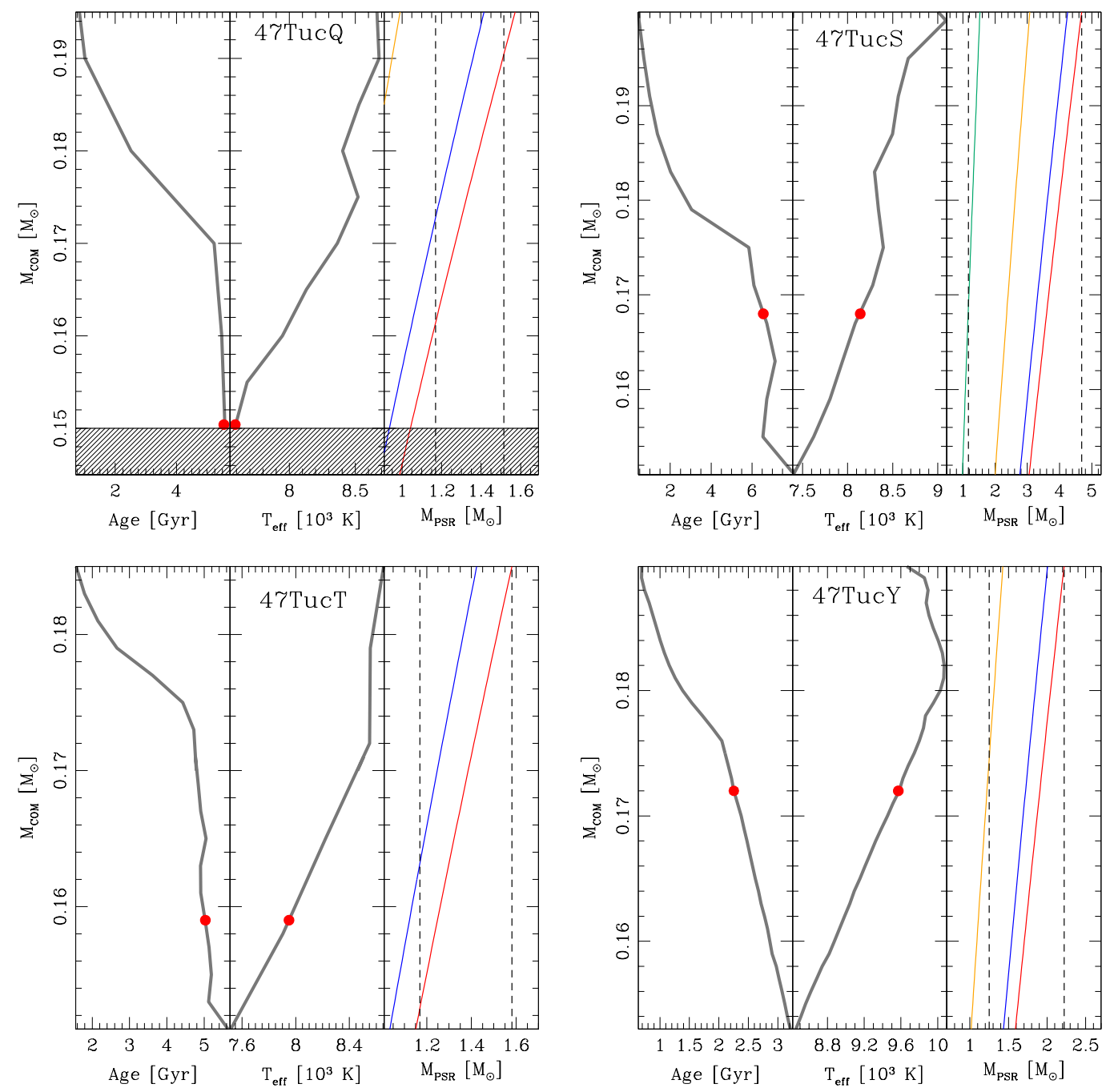

Fig. 4.- Physical properties of 47TucQ, 47TucS, 47TucT and 47TucY (see labels), as derived from the comparison between the photometric characteristics of each companion and the WD cooling track models. In each plot, the gray lines drawn in the left and central panels show the allowed combinations between companion mass and cooling age or temperature (see text). The red dots correspond to the most probable values. In the case of COM-47TucQ the shaded areas mark the region $\left(\mathrm{M}_{\mathrm{COM}}<0.15 \mathrm{M}_{\odot}\right)$ not sampled by the theoretical cooling tracks. In the rightmost panel of each plot, the solid curves represent the combination of values allowed by the PSR mass function for different inclination angles $\left(i=90^{\circ}\right.$ in red, $i=70^{\circ}$ in blue, $i=50^{\circ}$ in orange, and $i=30^{\circ}$ in green). The blue dashed lines correspond to the assumed minimum NS mass $\left(\sim 1.17 M_{\odot}\right.$; Janssen et al. 2008) and the largest NS mass value obtained for $i=90^{\circ}$. 


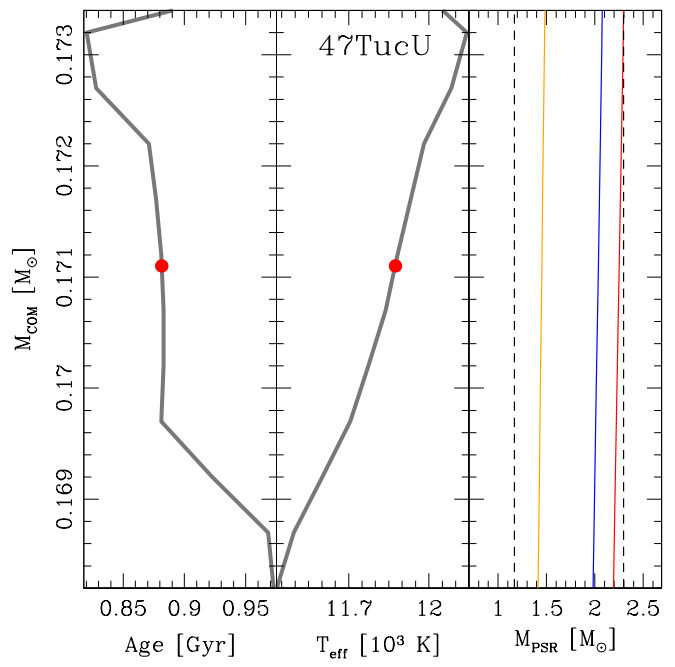

Fig. 5.- As in Fig. 4, but for 47TucU. 


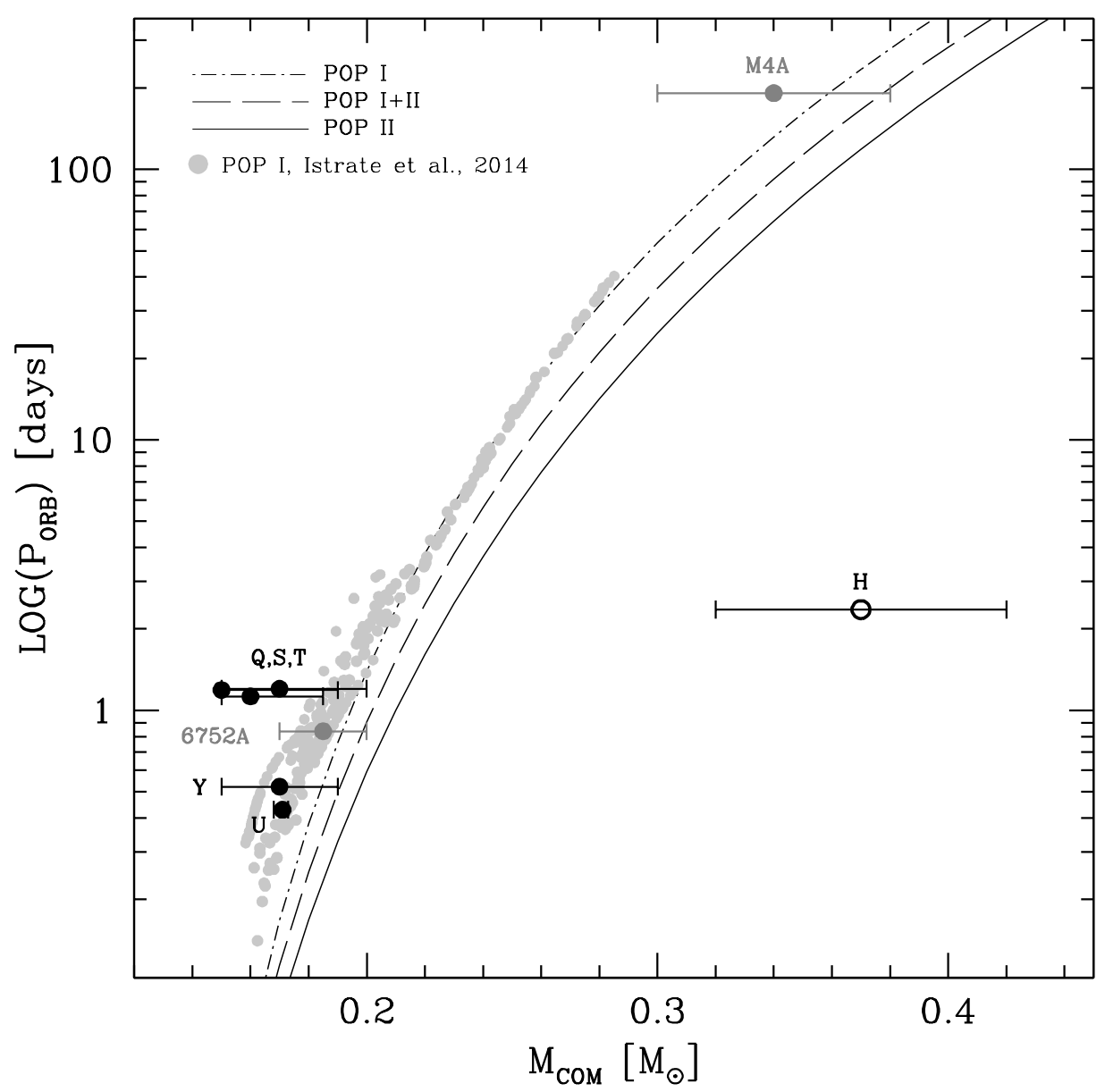

Fig. 6.- MSP orbital periods plotted as a function of the best-fit companion masses, for each identified object (see lables), plus the ones detected in NGC 6752 and M4 (dark gray points; see Ferraro et al. 2003a; Sigurdsson et al. 2003). The three curves correspond to the theoretical predictions of Tauris \& Savonije (1999) for three different stellar population progenitors, as reported in the top-left legend. The light gray points correspond to the theoretical results obtained by Istrate et al. (2014). 


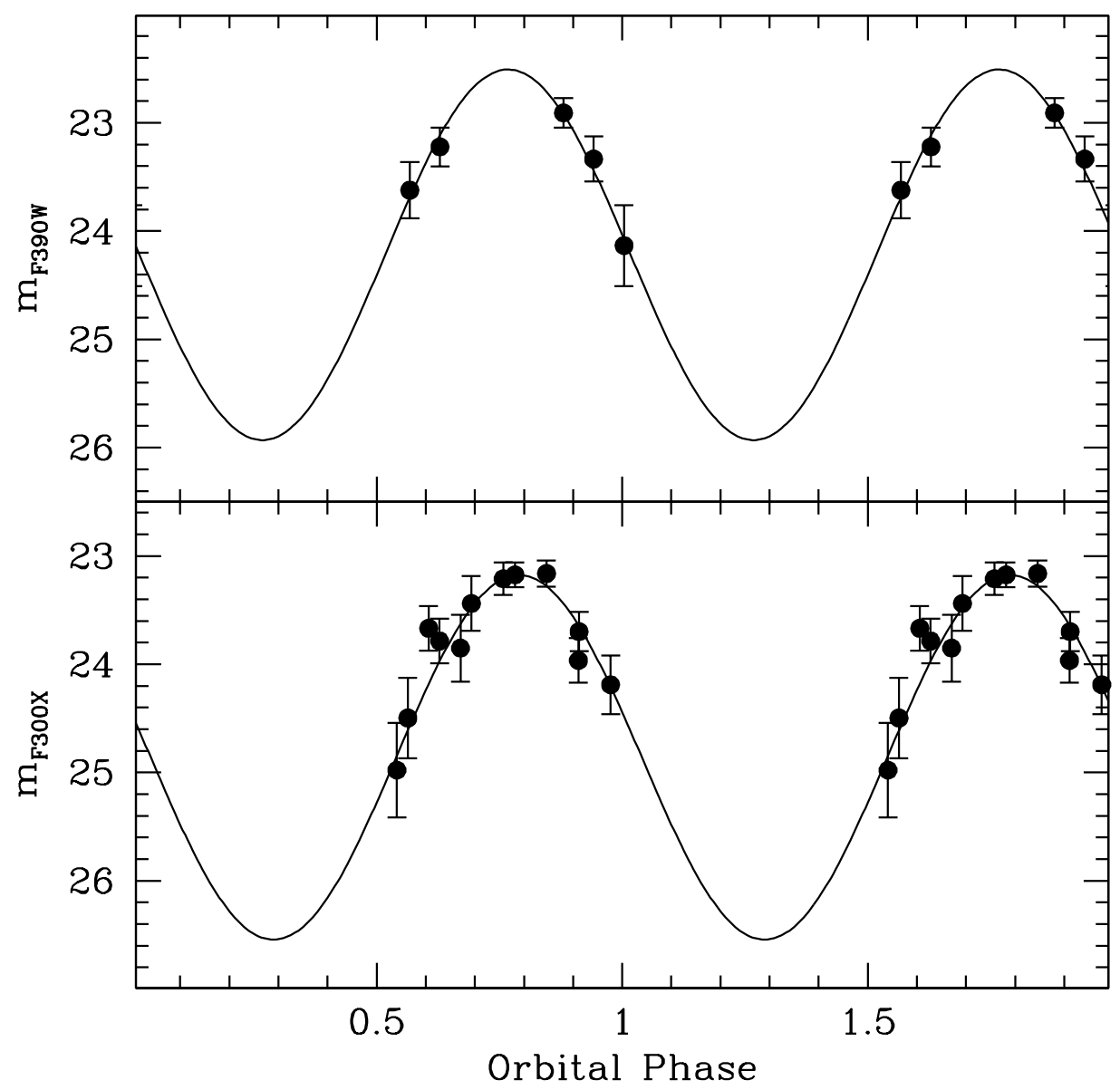

Fig. 7.- Light curves of COM-47TucW in the F390W (upper panel) and F300X (lower panel). The two curves are folded with the radio parameters and two periods are shown for clarity. The black curve in each panel is the best analytical model obtained independently for each filter. 